Bull. Austral. Math. Soc.

05D05, 05D99

VOL. $61(2000)$ [175-176]

\title{
Extremal problems in finite sets
}

\section{Paulette Lieby}

The main concern of this thesis is the study of problems involving collections of subsets of a finite set $[n]=\{1,2, \ldots, n\}$ and especially antichains on $[n]$. An antichain is a collection of sets in which no two sets are comparable under set inclusion. An antichain $\mathcal{A}$ is flat if there exists an integer $k \geqslant 0$ such that every set in $\mathcal{A}$ has cardinality either $k$ or $k+1$. The size of $\mathcal{A}$ is $|\mathcal{A}|$ and the volume of $\mathcal{A}$ is $\sum_{A \in \mathcal{A}}|A|$.

A unifying problem in the thesis is the flat antichain conjecture which states that $\mathcal{A}$ is an antichain on $[n]$ if and only if there exists a flat antichain on $[n]$ with the same size and volume as $\mathcal{A}$. The truth of the conjecture would provide a simple test for the existence of antichains on $[n]$ with a given size and volume. The flat antichain conjecture is known to hold in several special cases. This thesis shows that it holds in several further cases.

Two main approaches have been taken in the investigation of the flat antichain conjecture. The first approach consists of studying the volumes of antichains. Building on earlier work of Clements [1] we observe that for given $n$ and $s$, the antichains on $[n]$ of size $s$ which achieve minimum (maximum) volume are flat antichains, where the minimum (maximum) is taken over all antichains on $[n]$ of size $s$. Further, we show that if $\mathcal{A}$ is an antichain on $[n]$ then there exists a flat antichain on $[n]$ with the same volume as $\mathcal{A}$. In proving this last result we prove that the flat antichain conjecture holds in one special case.

The second approach involves counting the number of subsets and supersets of certain collections of sets as defined below. The squashed (or colex) order on sets is a set ordering with the property that the number of subsets of a collection of $k$-sets is minimised when the collection consists of an initial segment of $k$-sets in squashed order. In the universal set [n], consider the collections $L_{k+1}(p)$ and $L_{k-1}(p)$ consisting of the last $p$ $(k+1)$-sets in squashed order and the last $p(k-1)$-sets in squashed order respectively. Let $a_{1}$ be the number of supersets of size $k$ of the sets in $L_{k-1}(p)$. Let $a_{2}$ be the number of subsets of size $k$ of the sets in $L_{k+1}(p)$ which are not subsets of any $(k+1)$-set preceding the sets in $L_{k+1}(p)$ in squashed order. We show that $a_{1}+a_{2}>2 p$. This result, called

Received 21st September, 1999

Thesis submitted to the Northern Territory University, February 1999. Degree approved, September 1999. Supervisor: Dr. Ian Roberts.

Copyright Clearance Centre, Inc. Serial-fee code: $0004-9727 / 00 \quad \$ A 2.00+0.00$. 
the 3-levels result, enables us to show that the flat antichain conjecture holds in several cases.

Several conjectures and open problems which are related to the 3-levels result are stated and implications of the truth of the flat antichain conjecture are considered.

\section{REFERENCES}

[1] G.F. Clements, 'The minimal number of basic elements in a multiset antichain', J. Combin. Theory Ser. A 25 (1978), 153-162.

Faculty of Science

Northern Territory University

Casuarina NT 0909

Australia 